\title{
Stress and dysmenorrhoea: a population based prospective study
}

\author{
L Wang, X Wang, W Wang, C Chen, A G Ronnennberg, W Guang, A Huang, Z Fang, T Zang, \\ L Wang, $X$ Xu
}

See end of article for authors' affiliations .......................

Correspondence to: Dr X Wang, The Mary Ann and J. Milburn Smith Child Health Research Program, Children's Memorial Hospital, 2300 Children's Plaza, Box 157, Chicago, IL 60614-3394, USA: xbwang@ childrensmemorial.org

Accepted 10 August 2004
Background: Dysmenorrhoea is the most common gynaecological disorder in women of reproductive age. Despite the association between stress and pregnancy outcomes, few studies have examined the possible link between stress and dysmenorrhoea.

Aims and Methods: Using a population based cohort of Chinese women, the independent effect of women's perceived stress in the preceding menstrual cycle on the incidence of dysmenorrhoea in the subsequent cycle was investigated prospectively. The analysis included 1160 prospectively observed menstrual cycles from 388 healthy, nulliparous, newly married women who intended to conceive. The perception of stress and the occurrence of dysmenorrhoea in each menstrual cycle were determined from daily diaries recorded by the women.

Results: After adjustment for important covariates, the risk of dysmenorrhoea was more than twice as great among women with high stress compared to those with low stress in the preceding cycle $(O R=2.4 ; 95 \% \mathrm{Cl}$ 1.4 to 4.3 ). The risk of dysmenorrhoea was greatest among women with both high stress and a history of dysmenorrhoea compared to women with low stress and no history of dysmenorrhoea $(\mathrm{OR}=10.4,95 \% \mathrm{Cl}$ 4.9 to 22.3). Stress in the follicular phase of the preceding cycles had a stronger association with dysmenorrhoea than stress in the luteal phase of the preceding cycles.

Conclusion: This study shows a significant association between stress and the incidence of dysmenorrhoea, which is even stronger among women with a history of dysmenorrhoea.
D ysmenorrhoea, or painful menses, is the most common gynaecological disorder in women of reproductive age. The reported prevalence of primary dysmenorrhoea, which occurs in the absence of organic pelvic lesions, ranges from $43 \%$ to $90 \%$ among various populations. ${ }^{1-5}$ Approximately $10-15 \%$ of women have severe, disabling dysmenorrhoea, which can contribute to school absenteeism, lost work time, and reduced quality of life. ${ }^{467}$

There is growing evidence of an association between various measures of psychosocial stress and adverse pregnancy outcomes, such as preterm delivery. ${ }^{8-13}$ However, only a few studies to date have examined the relation between stress and the risk of dysmenorrhoea, ${ }^{14}{ }^{15}$ perhaps because of the methodological challenges inherent in studying this association. Stress, for instance, can be defined in various ways and is difficult to quantify, and dysmenorrhoea is a subjective condition that relies on self report. These features are particularly problematic in retrospective and crosssectional studies, in which the validity of stress and dysmenorrhoea data depends on a subject's ability to recall both events accurately. Such studies are also limited because a temporal relation between stress and dysmenorrhoea cannot be established, and causal inference is therefore impossible.

Using data from a large, population based prospective study of reproductive health among women textile workers in Anhui, China, we investigated whether a woman's perceived stress in one menstrual cycle was independently associated with the risk of dysmenorrhoea in the following cycle. By having women record the occurrence of both stress and dysmenorrhoea in daily diaries, we were able to reduce recall bias and explore the temporal relation between stress and dysmenorrhoea.

\section{MATERIALS AND METHODS \\ Subjects}

The current study is a part of a prospective reproductive health study among female workers at a textile mill located in Anqing city, Anhui Province, China. Anhui province has a rapid economy growth and a population of about 60 million. Those female workers engage in rotating shift work and their duties include weaving, twisting, and sewing. The major job related exposures include shift work, and dust and noise exposure. Average noise is about $90 \mathrm{db}$ in their workplace. The study protocols have been approved by the Human Subject Committee of the Chinese institutions involved in the study and by the Institutional Review Board of the Harvard School of Public Health.

The eligibility criteria for the field enrolment were as follows: (1) full time employed women workers; (2) newly married; (3) aged 20-34 years; and (4) had obtained permission to have a child (according to China's Family Planning Policy, each couple is allowed to have only one child). All women were nulliparous. Women were excluded if: (1) they were already pregnant before enrolment; (2) they had tried unsuccessfully to become pregnant for at least one year at any time in the past; and (3) they planned to quit/ change jobs or to move out of the city over the one year course of follow up.

A detailed description of field data collection can be found elsewhere. ${ }^{16}$ In brief, after obtaining informed consent, an interviewer administered a baseline questionnaire that collected historical data on menstruation, contraceptive use, reproductive history, sociodemographic characteristics, active

Abbreviations: $\mathrm{OR}$, odds ratio; $\mathrm{Cl}$, confidence interval; $\mathrm{SD}$, standard deviation; $\mathrm{BMI}$, body mass index 


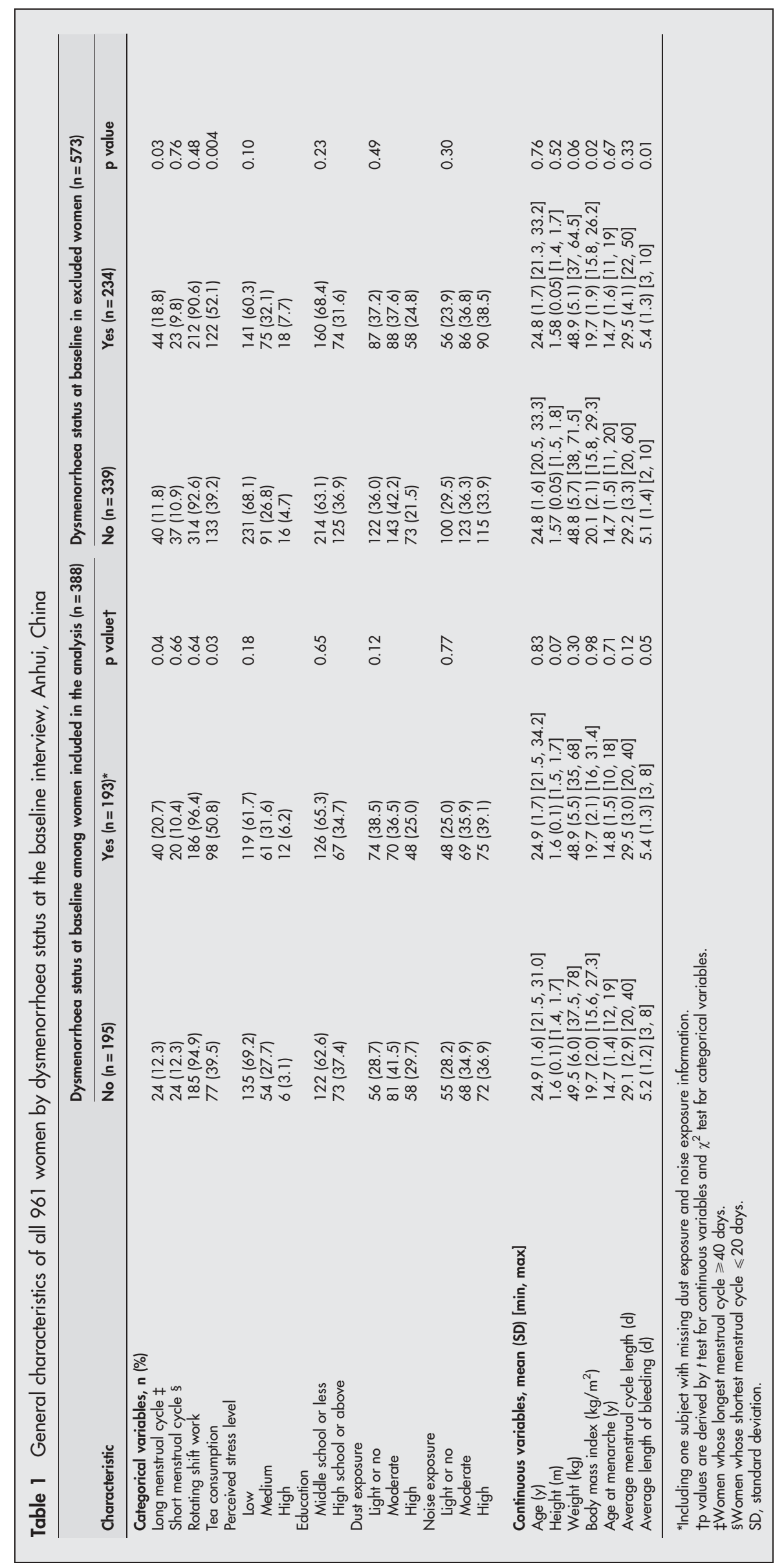


Table 2 Odds ratio (OR) and 95\% confidence interval (CI) for dysmenorrhoea in association with self perceived stress during the preceding menstrual cycle in 388 Chinese women

\begin{tabular}{|c|c|c|c|}
\hline $\begin{array}{l}\text { Stress in the } \\
\text { preceding cycles }\end{array}$ & $\begin{array}{l}\text { Total cyclest } \\
\text { (\% dysmenorrhoea) }\end{array}$ & OR $\ddagger$ & $95 \% \mathrm{Cl}$ \\
\hline Low & $494(21.9)$ & $1.0 \S$ & - \\
\hline Medium & $495(28.7)$ & 1.2 & 0.8 to 1.7 \\
\hline High & $171(43.9)$ & 2.4 & 1.4 to $4.3^{\text {** }}$ \\
\hline
\end{tabular}

${ }^{* *} p<0.01$.

†1160 cycles chosen from 388 subjects were included in this analysis. \$The outcome was defined as a binary variable adjusted for age, BMI, shift work, dust exposure, cycle length, education, and passive smoking. §Reference group.

smoking and passive smoke exposure, alcohol use, and environmental and occupational exposures, such as exposure to dust and noise, and perceived work stress. Beginning on the date when use of contraceptive methods was stopped, each woman kept a daily diary to record vaginal bleeding, menstrual pain, sexual intercourse, use of medications, and medical conditions. The daily diary was collected for up to 12 months or until a pregnancy was clinically confirmed.

Of the 971 women textile workers who met the eligibility criteria, 961 were enrolled and provided baseline information and 10 declined to participate. Of those, 388 women had necessary data for this study, including stress exposure information in the preceding cycle and complete baseline and daily diary information, and therefore were included in the analysis.

\section{Assessment of stress}

A menstrual cycle was defined as the period of time beginning with the first day of menstrual bleeding and continuing until the day immediately preceding the next menstrual bleeding period. For each prospectively observed menstrual cycle, women recorded their perceived general stress, representing both work stress and stress from other sources, in the daily diary. Each woman recorded her perceived stress in response to the question: "How would you describe your level of stress?" Response choices were: "(1) not stressful, (2) a little stressful, and (3) very stressful". Daily stress was correspondingly coded as low, medium, or high. In the analysis, cycle specific stress was defined for three time periods: (1) during the entire menstrual cycle; (2) during the follicular phase (from the first day of the menstrual cycle until the first day of the luteal phase); and (3) during the luteal phase of the cycle (two weeks prior to the first day of the next menses). Within each time period, "high stress" was defined as one or more days of reported high stress; "medium stress" was defined as one or more days of reported medium stress but no reported high stress; and "low stress" was defined as no reported high or medium stress. The cycle specific stress variables were defined as categorical variables and coded into low, medium, and high stress groups. These stress variables were used to predict the occurrence of dysmenorrhoea in the subsequent menstrual cycles.

\section{Assessment of dysmenorrhoea}

Menstrual pain was defined as abdominal or low back pain during menstrual bleeding (tong jing in Chinese). Dysmenorrhoea was defined as two or more days of menstrual pain within a menstrual cycle. Dysmenorrhoea for each prospectively observed menstrual cycle was based on daily diary records in which women recorded the occurrence of menstrual pain (yes or no). Past history of dysmenorrhoea was obtained from the baseline questionnaire in response to the question: "Did you experience dysmenorrhoea during your menses during the past 12 months?".

\section{Statistical analysis}

We assessed the risk of dysmenorrhoea in a prospectively observed menstrual cycle according to the level of general stress in the preceding menstrual cycle. We also estimated the association between stress and the odds of dysmenorrhoea based on the level of stress in both the follicular and luteal phases. Women were categorised into one of four groups. Group 1 consisted of women with low stress in both phases; group 2 included those with low stress in the follicular phase but medium or high stress in luteal phase; group 3 included women with medium or high stress in the follicular phase but low stress in the luteal phase; and group 4 consisted of women with medium or high stress level in both phases.

Logistic regression was used to estimate odds ratios (OR) and 95\% confidence intervals (CI) of dysmenorrhoea associated with stress, while adjusting for age, BMI, education, shift work, dust exposure, preceding menstrual cycle length, and passive smoking. The method of generalised estimating equations (GEE) was used to account for autocorrelations in the data arising from multiple observed menstrual cycles per subject. We also examined the potential interaction between stress and a history of dysmenorrhoea on the risk of dysmenorrhoea in subsequent cycles using two approaches: (1) we compared the risk of dysmenorrhoea associated with the stress variables, stratified by the presence or absence of a history of dysmenorrhoea; and (2) we also performed a likelihood ratio test for the interaction of these

Table 3 Odds ratio (OR) and 95\% confidence interval (Cl) for dysmenorrhoea in association with self perceived stress during different time phases of the preceding menstrual cycle

\begin{tabular}{|c|c|c|c|c|}
\hline $\begin{array}{l}\text { Stress in the } \\
\text { follicular phase of the } \\
\text { preceding cyclest }\end{array}$ & $\begin{array}{l}\text { Stress in the } \\
\text { luteal phase of the } \\
\text { preceding cyclest }\end{array}$ & $\begin{array}{l}\text { Total cycles } \\
\text { (\% dysmenorrhoea) }\end{array}$ & OR‡§ & $95 \% \mathrm{Cl} \S$ \\
\hline Low & Low & $738(24.5)$ & $1.0^{\circ}$ & - \\
\hline Low & Medium/high & 60 (31.7) & 1.4 & 0.8 to 2.5 \\
\hline Medium/high & & $42(42.9)$ & 2.2 & 1.1 to $4.5^{*}$ \\
\hline Medium/high & Medium/high & $49(65.3)$ & 6.4 & 2.5 to $15.9^{* * *}$ \\
\hline \multicolumn{5}{|c|}{$\begin{array}{l}{ }^{*} \mathrm{p}<0.05 ;{ }^{* * *} \mathrm{p}<0.001 \text {. } \\
+ \text { Exclude cycles with cycle length less than } 21 \text { days. } \\
\text { tThe outcome was defined as a binary variable adjusted for age, BMl, shift work, dust exposure, cycle length, } \\
\text { education, and passive smoking. } \\
\text { §OR, odds ratio; } \mathrm{Cl} \text {, confidence interval. } \\
\text { 'Reference group. }\end{array}$} \\
\hline
\end{tabular}


Table 4 Odds ratio (OR) and 95\% confidence interval (CI) for dysmenorrhoea in association with self perceived stress in the preceding menstrual cycle and a reported history of dysmenorrhoea at baseline

\begin{tabular}{|c|c|c|c|c|}
\hline $\begin{array}{l}\text { History of } \\
\text { dysmenorrhoea }\end{array}$ & $\begin{array}{l}\text { Stress in the } \\
\text { preceding cycles }\end{array}$ & $\begin{array}{l}\text { Total cycles } \\
\text { (\% dysmenorrhoea) }\end{array}$ & OR†§ & $95 \% \mathrm{Cl} \S$ \\
\hline No & Low & $257(10.9)$ & 1.0 & - \\
\hline No & Medium & 243 (17.3) & 1.3 & 0.7 to 2.2 \\
\hline No & High & 73 (24.7) & 2.1 & 0.9 to 5.2 \\
\hline Yes & Low & $237(33.8)$ & 4.2 & 2.3 to $7.6^{* * *}$ \\
\hline Yes & Medium & $252(39.7)$ & 4.6 & 2.6 to $8.3^{* * *}$ \\
\hline Yes & High & $98(58.2)$ & 10.4 & 4.9 to $22.3^{* * *}$ \\
\hline \multicolumn{5}{|c|}{$\begin{array}{l}\text { *** }<0.001 \text {. } \\
\text { †The outcome was defined as a binary variable adjusted for age, BMI, shift work, dust exposure, cycle length, } \\
\text { education, and passive smoking. } \\
\text { łHistory of dysmenorrhoea refers to experience of dysmenorrhoea within a year prior to enrolment, obtained from } \\
\text { baseline questionnaire. } \\
\S O R \text {, odds ratio; Cl, confidence interval. } \\
\text { ๆReference group. }\end{array}$} \\
\hline
\end{tabular}

two factors using an additive model that assessed the odds of dysmenorrhoea. All analyses were performed using SAS software version 8.02 (SAS Institute, Inc., Cary, North Carolina).

\section{RESULTS}

This analysis includes 1160 prospectively observed menstrual cycles from 388 women with stress exposure information in the preceding cycle and with complete baseline and daily diary information. The overall prevalence of dysmenorrhoea is $44.4 \%$. Table 1 compares characteristics of the 388 women included and 573 women excluded from our analysis by dysmenorrhoeal status at the baseline. There were no significant differences between those women included and excluded in terms of baseline characteristics, including age, perceived stress level, education, height, weight, body mass index, age at menarche, and occupational exposures. Women with dysmenorrhoea were more likely to have long menstrual cycles and to drink tea. Among the 388 women included in the analysis, the overall proportions of women with low, medium, and high perceived stress level are $65.5 \%, 30.0 \%$, and $5.0 \%$ respectively, but the proportion of medium and high stress was higher among women with dysmenorrhoea.

The overall incidence of dysmenorrhoea was $28 \%$ among 1160 prospectively observed menstrual cycles. The incidence of dysmenorrhoea increased with increasing levels of perceived stress and was $21.9 \%$ for women with low stress, $28.7 \%$ for those with medium stress, and $43.9 \%$ for women with high stress (table 2). After adjusting for potentially confounding variables, the relative odds of dysmenorrhoea was 2.4 (95\% CI 1.4 to 4.3 ) for women with high stress in the previous cycle compared to those with low stress. We also assessed whether the phase of the menstrual cycle in which stress was recorded influenced the risk of dysmenorrhoea in the subsequent cycle (table 3 ). Compared to women with low stress in both the follicular and luteal phases of the previous cycle, the adjusted OR for dysmenorrhoea was 2.2 (95\% CI 1.1 to 4.5 ) for women with stress in the follicular phase only and 6.4 (95\% CI 2.5 to 15.9) for those with stress in both the follicular and luteal phases.

We used a stratified analysis to investigate whether the association between stress and dysmenorrhoea differed between subgroups of cycles with or without dysmenorrhoea in the preceding cycles (data not shown). The OR of dysmenorrhoea associated with high stress was 3.6 (95\% CI 1.7 to 7.8 ) among cycles with dysmenorrhoea in the preceding cycle and 1.3 (95\% CI 0.7 to 2.7 ) for those without dysmenorrhoea in the preceding cycle.
When we examined the combined effects of a history of dysmenorrhoea (from the baseline questionnaire) and stress in the preceding menstrual cycle, both factors contributed to an increased risk of dysmenorrhoea in the subsequent cycle (table 4). This combined effect is most pronounced among women with both high stress in the preceding cycle and a history of dysmenorrhoea, for whom the risk of dysmenorrhoea in the subsequent cycle was more than nine-fold higher than for women with low stress and no history of dysmenorrhoea $(\mathrm{OR}=10.4,95 \%$ CI 4.9 to 22.3$)$. We also assessed the interaction between stress in the preceding cycle and a history of dysmenorrhoea on the odds of dysmenorrhoea. The statistical significance of this interactive effect was tested with the $\mathrm{z}$ statistic as described by Hogan and colleagues. ${ }^{17}$ Our result suggested a possible interaction between the two factors on an additive scale, although it was not statistically significant $(z=1.325, \mathrm{p}=0.185)$.

\section{DISCUSSION}

Using a prospective, population based cohort of Chinese women, we found significant dose-response and temporal associations between perceived stress in one menstrual cycle and the occurrence of dysmenorrhoea in the subsequent cycle. We also found that, compared to low stress throughout the cycle, perceived stress in the follicular phase of the menstrual cycle appeared to have a greater influence on subsequent dysmenorrhoea than did stress in the luteal phase; stress during both phases was associated with the highest risk of dysmenorrhoea in the following cycle. A history of dysmenorrhoea, either in the preceding cycle or as reported at baseline, appeared to strengthen the association between stress and dysmenorrhoea in the subsequent cycle.

The observed association between stress and risk of dysmenorrhoea is consistent with previous reports. ${ }^{14}{ }^{15}$ In a study of women textile workers in China, Christiani et al reported that, compared to women with low stress, the risk of dysmenorrhoea was $60 \%$ greater among women with moderate stress and more than twice as great among those with high stress. ${ }^{14}$ Gordley et al obtained similar results in a study of US Air Force employees in which high life stress was associated with a more than twofold increase in risk for dysmenorrhoea. ${ }^{15}$ Clarvit, however, did not find an association between stress and dysmenorrhoea in a group of medical students, which suggests that the relation between stress and dysmenorrhoea may differ among groups of women. ${ }^{18}$

The observed association between stress and dysmenorrhoea is biologically plausible although the biological mechanism(s) that link stress to dysmenorrhoea are not 
completely understood. When persons are under internal and/or external stress, they undergo a cascade of neuroendocrine responses. Corticotrophin releasing hormone (CRH), the major hypothalamic regulator of the mammalian stress response, mediates pituitary adrenocorticotrophic hormone (ACTH) secretion; the latter enhances adrenal cortisol secretion. ${ }^{19}{ }^{20}$ Stress is also known to inhibit the pulsatile release of follicle stimulating hormone and luteinising hormone, leading to impaired follicular development. ${ }^{21}$ Because synthesis of progesterone is increased in the luteinised follicle following ovulation, stress induced impairment of follicular development could potentially alter progesterone synthesis and release. Progesterone is thought to play an important role in dysmenorrhoea. Menstrual pain occurs only in ovulatory cycles, and progesterone has been shown to affect both the synthesis of prostaglandins $\mathrm{PGF}_{2 \alpha}$ and $\mathrm{PGE}_{2}{ }^{6}$ and the binding of these prostaglandins to myometrial receptors. ${ }^{1}$ Prostaglandins affect uterine muscle and vascular tone, ${ }^{722-24}$ and an imbalance of prostaglandins has been linked to the occurrence of dysmenorrhoea. ${ }^{1}$ Besides progesterone, stress related hormones, including adrenaline ${ }^{1025}$ and cortisol, ${ }^{2627}$ also appear to influence prostaglandin synthesis, which suggests that stress may have both direct and secondary effects on prostaglandin concentrations in the myometrium.

Our study has several strengths. Its prospective design helps eliminate potential recall bias, which is an inherent weakness of retrospective and cross-sectional studies. In addition, our subjects constituted a remarkably homogeneous cohort. Because all women were nulliparous and intended to conceive, our results were not influenced by parity or use of oral contraceptives, which have been associated with reduced menstrual pain, ${ }^{28}$ or by the use of intrauterine devices, which may complicate diagnosis of primary dysmenorrhoea. ${ }^{29}$ Although previous studies have shown an association between dysmenorrhoea and cigarette smoking, ${ }^{30}$ all of our subjects were non-smokers. In addition, our analyses adjusted for many potential environmental and occupational exposures, such as passive smoke exposure, that have been associated with dysmenorrhoea. ${ }^{31}$ The doseresponse and temporal relations we observed in this cohort, coupled with plausible potential biological mechanisms, suggest a causal association between stress and dysmenorrhoea.

Despite its strengths, several limitations of our study should be considered. Exposure to stress was based on a self recorded daily diary and not on responses to a standardised instrument. Self recorded stress is by its nature subjective and, therefore, susceptible to recall bias. Moreover, we did not have detailed information on stress exposure at work versus stress at home; we could not, therefore, fully assess the separate effects of the stress source. Another potential limitation concerns the fact that dysmenorrhoea is diagnosed based solely on a woman's perception of pain, which is difficult to quantify and could be related to non-menstrual events. However, because this is a young and healthy cohort, the likelihood of non-menstrual pain or secondary dysmenorrhoea is very small. Although the homogeneity of our cohort helps to simplify interpretation of the relation between stress and dysmenorrhoea, it also somewhat limits our ability to apply our findings to other populations where, for instance, women may not be employed or where smoking and use of alcohol may be more common. Another concern is that about $40 \%$ of all the eligible women had complete information and thus were included in this analysis. However, as table 1 showed, women who were included in the analysis and those excluded did not differ significantly in baseline demographic characteristics and in the prevalence of stress and dysmenorrhoea.
Identification of modifiable risk factors for dysmenorrhoea is important because the condition affects a large proportion of women of reproductive age and contributes to school absenteeism, lost work time, and reduced quality of life. ${ }^{467}$ Stress reduction programmes aimed at reducing both personal and job related stress by enhancing control and social support may help to reduce the occurrence of dysmenorrhoea in textile workers. ${ }^{32}$ Prevention may be a safer approach to dysmenorrhoea than pharmacological treatment, particularly among women who are attempting to become pregnant, because most of the non-steroidal antiinflammatory drugs commonly used to treat dysmenorrhoea are generally contraindicated during pregnancy. ${ }^{33}{ }^{34}$ Stress reduction programmes aimed at reproductive age women, especially those with a history of dysmenorrhoea, may be considered as possible preventive strategies to reduce the occurrence of dysmenorrhoea as well as the resulted absenteeism and reduced work productivity.

\section{Authors' affiliations}

Lin Wang, W Wang, C Chen, A G Ronnennberg, X Xu, Department of Environmental Health, Harvard School of Public Health, Boston, MA, USA

X Wang, The Mary Ann and J. Milburn Smith Child Health Research Program, Children's Memorial Hospital, Chicago, IL, USA

W Guang, A Huang, Z Fang, T Zang, Institute for Biomedicine, Anhui Medical University, Anhui, China

Lihua Wang, Department of Environmental Health, School of Public Health, Peking University, Beijing, China

This study is supported in part by grants 1R01 HD32505 from the National Institute of Child Health and Human Development; 1RO1 ES08337, ES-00002, P01 ES06198, and 1R01 ES11682 from the National Institute of Environmental Health Science; and 20-FY98-0701 and 20-FY02-56 from the March of Dimes Birth Defects Foundation, USA

Please address requests for reprints to: $\operatorname{Dr} X X_{U}$, Department of Environmental Health, FXB101, Harvard School of Public Health, 665 Huntington Avenue, Boston, MA 02115, USA; xu@hsph.harvard.edu

\section{REFERENCES}

1 Ylikorkala O, Dawood MY. New concepts in dysmenorrhea. Am J Obstet Gynecol 1978;130:833-47.

2 Svanberg L, Ulmsten U. The incidence of primary dysmenorrhea in teenagers. Arch Gynecol 1981;230:173-7.

3 Andersch B, Milsom I. An epidemiologic study of young women with dysmenorrhea. Am J Obstet Gynecol 1982;144:655-60.

4 Harlow SD, Park M. A longitudinal study of risk factors for the occurrence, duration and severity of menstrual cramps in a cohort of college women. Br J Obstet Gynaecol 1996; 103: 1 134-42

5 Jamieson DJ, Steege JF. The prevalence of dysmenorrhea, dyspareunia, pelvic pain, and irritable bowel syndrome in primary care practices. Obstet Gynecol 1996;87:55-8.

6 Friederich MA. Dysmenorrhea. Women Health 1983;8:91-106.

7 Dawood MY. Dysmenorrhea. J Reprod Med 1985;30:154-67.

8 Lobel M, Dunkel-Schetter C, Scrimshaw SC, et al. Prenatal maternal stress and prematurity: a prospective study of socioeconomically disadvantaged women. Health Psychol 1992;11:32-40.

9 Copper RL, Goldenberg RL, Das A, et al. The preterm prediction study: maternal stress is associated with spontaneous preterm birth at less than thirtyfive weeks' gestation. National Institute of Child Health and Human Development Maternal-Fetal Medicine Units Network. Am J Obstet Gynecol 1996; 175:1286-92

10 Hedegaard M, Henriksen TB, Secher NJ, et al. Do stressful life events affect duration of gestation and risk of preterm delivery? Epidemiology 1996;7:339-45.

11 Nordentoft M, Lou HC, Hansen D, et al. Intrauterine growth retardation and premature delivery: the influence of maternal smoking and psychosocial factors. Am J Public Health 1996;86:347-54.

12 Rini CK, Dunkel-Schetter C, Wadhwa PD, et al. Psychological adaptation and birth outcomes: the role of personal resources, stress, and sociocultural context in pregnancy. Health Psychol 1999;18:333-45.

13 Dole N, Savitz DA, Hertz-Picciotto I, et al. Maternal stress and preterm birth. Am J Epidemiol 2003;157:14-24.

14 Christiani DC, Niu T, Xu X. Occupational stress and dysmenorrhea in women working in cotton textile mills. Int J Occup Environ Health 1995; 1:9-15. 
15 Gordley LB, Lemasters G, Simpson SR, et al. Menstrual disorders and occupational, stress, and racial factors among military personnel. J Occup Environ Med 2000;42:871-81.

16 Wang X, Chen C, Wang L, et al. Conception, early pregnancy loss, time to clinical pregnancy. Fertil Steril 2003;79:577-84.

17 Hogan MD, Kupper LL, Most BM, et al. Alternatives to Rothman's approach for assessing synergism (or antagonism) in cohort studies. Am J Epidemiol 1978;108:60-7.

18 Clarvit SR. Stress and menstrual dysfunction in medical students. Psychosomatics 1988;29:404-9.

19 Vale W, Spiess J, Rivier C, et al. Characterization of a 41-residue ovine hypothalamic peptide that stimulates secretion of corticotropin and betaendorphin. Science 1981;213:1394-7.

20 Wadhwa PD, Dunkel-Schetter C, Chicz-DeMet A, et al. Prenatal psychosocial factors and the neuroendocrine axis in human pregnancy. Psychosom Med 1996;58:432-46.

21 Chatterton RT. The role of stress in female reproduction: animal and human considerations. Int J Fertil 1990;35:8-13.

22 Willman EA, Collins WP, Clayton SG. Studies in the involvement of prostaglandins in uterine symptomatology and pathology. Br J Obstet Gynaecol 1976;83:337-41.

23 Lundstrom V, Green K. Endogenous levels of prostaglandin F2alpha and its main metabolites in plasma and endometrium of normal and dysmenorrheic women. Am J Obstet Gynecol 1978;130:640-6.

24 Rees MC, Anderson AB, Demers LM, et al. Prostaglandins in menstrual fluid in menorrhagia and dysmenorrhoea. Br J Obstet Gynaecol 1984;91:673-80.
25 Austin MP, Leader L. Maternal stress and obstetric and infant outcomes: epidemiological findings and neuroendocrine mechanisms. Aust N Z J Obstet Gynaecol 2000;40:331-7

26 Casey ML, MacDonald PC, Mitchell MD. Despite a massive increase in cortisol secretion in women during parturition, there is an equally massive increase in prostaglandin synthesis. A paradox? J Clin Invest 1985;75:1852-7.

27 Morimoto K, Oku M. [Effect of progesterone, cortisol and dehydroepiandrosterone-sulfate on prostaglandin production by cultured human myometrial cells]. Nippon Sanka Füinka Gakkai Zasshi 1995;47:391-7.

28 Sundell G, Milsom I, Andersch B. Factors influencing the prevalence and severity of dysmenorrhoea in young women. Br J Obstet Gynaecol 1990;97:588-94.

29 Kaunitz AM. Reappearance of the intrauterine device: a 'user-friendly' contraceptive. Int J Fertil Womens Med 1997;42:120-7.

30 Hornsby PP, Wilcox AJ, Weinberg CR. Cigarette smoking and disturbance of menstrual function. Epidemiology 1998;9:193-8.

31 Chen C, Cho SI, Damokosh Al, et al. Prospective study of exposure to environmental tobacco smoke and dysmenorrhea. Environ Health Perspect 2000;108:1019-22.

32 Levi L, Bartley M, Marmot $M$, et al. Stressors at the workplace: theoretical models. Occup Med 2000;15:69-106.

33 Ericson A, Kallen BA. Nonsteroidal anti-inflammatory drugs in early pregnancy. Reprod Toxicol 2001;15:371-5.

34 Hayes EC, Rock JA. COX-2 inhibitors and their role in gynecology. Obstet Gynecol Surv 2002;57:768-80. 